\title{
Tracing jet emission at the base of a high-mass YSO
} First AMBER/VLTI observations of the Bry emission in IRAS 13481-6124^

\author{
A. Caratti o Garatti ${ }^{1}$, B. Stecklum ${ }^{2}$, G. Weigelt ${ }^{3}$, D. Schertl ${ }^{3}$, K.-H. Hofmann ${ }^{3}$, S. Kraus ${ }^{4}$, R. D. Oudmaijer ${ }^{5}$, \\ W. J. de $\mathrm{Wit}^{6}$, A. Sanna ${ }^{3}$, R. Garcia Lopez ${ }^{1}$, A. Kreplin ${ }^{4}$, and T. P. Ray ${ }^{1}$ \\ 1 Dublin Institute for Advanced Studies, School of Cosmic Physics, Astronomy \& Astrophysics Section, 31 Fitzwilliam Place, \\ Dublin 2, Ireland \\ e-mail: alessio@cp.dias.ie \\ 2 Thüringer Landessternwarte Tautenburg, Sternwarte 5, 07778 Tautenburg, Germany \\ 3 Max-Planck-Institut für Radioastronomie, Auf dem Hügel 69, 53121 Bonn, Germany \\ 4 University of Exeter, School of Physics, Stocker Road, Exeter, EX4 4QL, UK \\ 5 School of Physics and Astronomy, University of Leeds, Leeds, LS2 9JT, UK \\ ${ }^{6}$ ESO-European Organisation for Astronomical Research in the Southern Hemisphere, Alonso de Cordova 3107, \\ Vitacura, Santiago de Chile, Chile
}

Received 27 January 2016 / Accepted 17 March 2016

\section{ABSTRACT}

\begin{abstract}
Aims. To probe the circumstellar environment of IRAS 13481-6124, a $20 M_{\odot}$ high-mass young stellar object (HMYSO) with a collimated parsec-scale jet and an accretion disc, we investigate the origin of its $\mathrm{Br} \gamma$ emission line through near-infrared (NIR) interferometry.

Methods. We present the first AMBER/VLTI observations of the Br $\gamma$ emitting region in an HMYSO at medium spectral resolution $(R=1500)$.

Results. Our AMBER/VLTI observations reveal a spatially and spectrally resolved Br $\gamma$ line in emission with a strong PCygni profile, indicating outflowing matter with a terminal velocity of $\sim 500 \mathrm{~km} \mathrm{~s}^{-1}$. Visibilities, differential phases, and closure phases are detected in our observations within the spectral line and in the adjacent continuum. Both total visibilities (continuum plus line emitting region) and pure-line visibilities indicate that the Br $\gamma$-emitting region is more compact (2-4 mas in diameter or $\sim 6-13$ au at $3.2 \mathrm{kpc}$ ) than the continuum-emitting region ( $\sim 5.4$ mas or $\sim 17 \mathrm{au})$. The absorption feature is also spatially resolved at the longest baselines (81 and $85 \mathrm{~m}$ ) and has a visibility that is slightly smaller than the continuum-emitting region. The differential phases at the four longest baselines display an " $\mathrm{S}$ "-shaped structure across the line, peaking in the blue- and red-shifted high-velocity components. The calibrated photocentre shifts are aligned with the known jet axis, i.e they are probably tracing an ionised jet. The high-velocity components $\left(v_{\mathrm{r}} \sim 100-500 \mathrm{~km} \mathrm{~s}^{-1}\right)$ are located far from the source, whereas the low-velocity components $\left(0-100 \mathrm{~km} \mathrm{~s}^{-1}\right)$ are observed to be closer, indicating a strong acceleration of the gas flow in the inner $10 \mathrm{au}$. Finally, a non-zero closure phase along the continuum is detected. By comparing our observations with the synthetic images of the continuum around $2.16 \mu \mathrm{m}$, we confirm that this feature originates from the asymmetric brightness distribution of the continuum owing to the inclination of the inner disc.
\end{abstract}

Key words. stars: formation - circumstellar matter - stars: protostars - stars: massive - techniques: interferometric techniques: high angular resolution

\section{Introduction}

The evolution of young stellar objects (YSOs), over a wide range of masses, seems to be characterised by significant accretion from a magnetised circumstellar disc and ejection of collimated jets. Both processes are tightly related: jets can remove excess angular momentum, so that some of the disc material can accrete onto the star (see, e.g. Königl \& Pudritz 2000; Pudritz et al. 2007). Their interplay is relatively well understood and tested in low-mass YSOs, whereas not much is known about high-mass YSOs (HMYSOs; $M>8 M_{\odot}$; O and early B spectral types), as their formation mechanism and evolution are still a matter of debate (see, e.g. Tan et al. 2014). Substantial progress in understanding the formation mechanism

\footnotetext{
* Based on observations collected at the VLT (ESO Paranal, Chile) with programmes 090.C-0371(B).
}

of HMYSOs is gained by connecting the gas dynamics close to the forming star (e.g. Kraus et al. 2010; Ilee et al. 2013; Cesaroni et al. 2014; Sanna et al. 2015) with the parsec-scale jet and outflow emission being driven by the central accreting source (e.g. Sanna et al. 2014; Caratti o Garatti et al. 2015a). Moreover, compelling observational evidence for the accretion disc scenario has recently come from the detection of compact discs in Keplerian rotation (e.g. Kraus et al. 2010; Ilee et al. 2013; Cesaroni et al. 2014) and parsec-scale collimated jets driven by HMYSOs (e.g. Stecklum et al. 2009; Varricatt et al. 2010; Caratti o Garatti et al. 2015a).

IRAS 13481-6124 $(\alpha(\mathbf{J} 2000)=13: 51: 37.856, \delta(\mathrm{J} 2000)=$ $-61: 39: 07.52)$ is among the few HMYSOs where an accretion disc (Kraus et al. 2010; Ilee et al. 2013; Boley et al. 2016), an outflow, and a collimated parsec-scale jet (Kraus et al. 2010; Stecklum et al. 2012; Caratti o Garatti et al. 2015a) have been 
Table 1. Log of the VLTI/AMBER observations of IRAS 13481-6124.

\begin{tabular}{ccccccccccc}
\hline \hline $\begin{array}{c}\text { IRAS 13481-6124 } \\
\begin{array}{c}\text { Observation } \\
\text { date }\end{array}\end{array}$ & $\begin{array}{c}\text { Time [UT] } \\
\text { Start }\end{array}$ & End & $\begin{array}{c}\text { Unit Telescope } \\
\text { array }\end{array}$ & $\begin{array}{c}\text { Spectral } \\
\text { mode }\end{array}$ & $\begin{array}{c}\text { Wavelength } \\
\text { range } \\
(\mu \mathrm{m})\end{array}$ & DIT $^{a}$ & $N^{b}$ & Seeing & Baseline & PA \\
\hline 2013 Feb. 28 & $08: 30$ & $08: 55$ & UT1-UT2-UT3 & MR-K-2.1 & $1.926-2.275$ & 0.3 & 3200 & $0.8-1.1$ & $40 / 46 / 85$ & $54 / 38 / 45$ \\
2013 Feb. 28 & $09: 11$ & $09: 42$ & UT1-UT2-UT3 & MR-K-2.1 & $1.926-2.275$ & 0.3 & 4800 & $0.6-0.9$ & $38 / 44 / 81$ & $62 / 45 / 53$ \\
\hline
\end{tabular}

Notes. ${ }^{(a)}$ Detector integration time per interferogram. ${ }^{(b)}$ Number of interferograms.

detected. Located at a distance of $\sim 3.2 \mathrm{kpc}$, IRAS 13481-6124 has a bolometric luminosity of $5.7 \times 10^{4} L_{\odot}$ (Lumsden et al. 2013). By modelling the spectral energy distribution (SED), Grave \& Kumar (2009) inferred an age of $\sim 10^{4} \mathrm{yr}$ and a protostellar mass of $\sim 20 M_{\odot}$ (i.e. an O9 ZAMS spectral type). Nearinfrared (NIR) interferometric observations with the Very Large Telescope Interferometer (VLTI) in the $K$-band continuum revealed a compact dusty disc ( $\sim 5.4$ mas in diameter, or $\sim 17.3 \mathrm{au}$ at $3.2 \mathrm{kpc}$; Kraus et al. 2010), tilted by $\sim 45^{\circ}$ with respect to the plane of the sky. Modelling of the CO band-head emission lines at $2.3 \mu \mathrm{m}$ suggests a disc in Keplerian rotation (Ilee et al. 2013). Perpendicular to the disc, a well-collimated (precession angle $\sim 8^{\circ}$ ) parsec-scale jet has also been detected (with position angle - PA - of $\sim 206^{\circ} / 26^{\circ}$ east of north, blue- and red-shifted lobes, respectively; Caratti o Garatti et al. 2015a), being traced by shocked $\mathrm{H}_{2}$ and [Fe II] lines. The shocked $\mathrm{H}_{2}$ emission is observed down to $\sim 20000$ au from the source (Stecklum et al. 2012). Closer to the source (a few arcseconds), the $\mathrm{H}_{2}$ emission is mostly excited by fluorescence and it is not detected at the smallest spatial scales observed with SINFONI $\left(\sim 0.1^{\prime \prime}\right.$ or $\sim 320 \mathrm{au}$; Stecklum et al. 2012). NIR spectroscopy also shows bright H I emission, confined to the HMYSO circumstellar environment ( $\leq 10000 \mathrm{au}$; Stecklum et al. 2012). The Br $\gamma$ line on source shows a shallow P Cygni profile and high-velocity wings (several hundred $\mathrm{km} \mathrm{s}^{-1}$; Stecklum et al. 2012). Their spectroastrometric analysis indicates a photocentre shift between line and continuum. The $\mathrm{Br} \gamma$ line profile might then be the product of different kinematical components, which originate from different locations: magnetospheric accretion flows, a hot disc atmosphere, strong winds from the stellar surface, or from the inner regions of the disc (within a few au), or an extended and collimated ionised jet.

To clarify the nature of the different kinematic components of the $\mathrm{Br} \gamma$ line and probe the circumstellar environment of IRAS 13481-6124, we therefore carried out, for the first time, VLTI/AMBER spectro-interferometric observations of this HMYSO at medium spectral resolution. This is the first NIR interferometric study that spatially and spectrally resolves an HMYSO in both continuum and Br $\gamma$ line.

Section 2 reports our interferometric observations and data reduction, while the interferometric results are presented in Sect. 3. Finally, in Sect. 4, we discuss the origin of the $\mathrm{Br} \gamma$ in IRAS 13481-6124.

\section{Observations and data reduction}

IRAS 13481-6124 was observed on the 28 February 2013 in two runs at the ESO/VLTI with AMBER (Petrov et al. 2007) and the UT1-UT2-UT3 telescope configuration. The projected baseline lengths extend from $\sim 38 \mathrm{~m}$ to $\sim 85 \mathrm{~m}$, and their position angle ranges between $\sim 38^{\circ}$ and $\sim 62^{\circ}$, i.e. relatively close to being parallel to the jet PA. Details of the observational settings are reported in Table 1.
We used AMBER medium spectral resolution mode in the $K$-band (MR-2.1 mode with nominal $R=1500$ ) covering the spectral range from 1.926 to $2.275 \mu \mathrm{m}$ around the $\mathrm{Br} \gamma$-line emission (at $2.166 \mu \mathrm{m}$ ). Although IRAS 13481-6124 in the $K$ band is bright enough ( $K=4.9 \mathrm{mag})$ to be observed interferometrically with AMBER and the UTs, its $H$-band magnitude ( $H=7.6 \mathrm{mag}$ ) exceeds the limit for employing the fringe tracker FINITO (Gai et al. 2004), which works in that band. The adopted detector integration time (DIT) was $0.3 \mathrm{~s}$ per interferogram and we integrated on source for about 25 and $30 \mathrm{~min}$ in the first and second run, respectively. Owing to the better average seeing of the second run $\left(\sim 0.75^{\prime \prime}\right.$ vs. $\left.\sim 0.95^{\prime \prime}\right)$ and the slightly longer exposure on source, the second-run data have a higher $\mathrm{S} / \mathrm{N}$ ratio. Star HD 103125 was observed before and after the science observations with the same observational settings, and used as an interferometric calibrator to derive the transfer function. To reduce our interferograms, we applied our own data reduction software, that is based on the P2VM algorithm (Tatulli et al. 2007) and that provides us with wavelength-dependent visibilities, wavelength-dependent differential phases, closure phases, and wavelength-calibrated spectra. The wavelength calibration was refined using the numerous telluric lines present in the observed wavelength range (for more details on the wavelength calibration method, see Weigelt et al. 2011; Garcia Lopez et al. 2015). We estimate an uncertainty in the wavelength calibration of $\sim 1.5 \AA\left(\sim 20 \mathrm{~km} \mathrm{~s}^{-1}\right)$. The spectral resolution measured on the spectrally unresolved telluric features around the $\mathrm{Br} \gamma$ line is $R \sim 2200$ or $\Delta v \sim 140 \mathrm{~km} \mathrm{~s}^{-1}$. To convert the observed wavelengths into radial velocities, we used a local standard of rest (LSR) velocity of $-37.9 \mathrm{~km} \mathrm{~s}^{-1}$ (Lumsden et al. 2013). Therefore all the velocities provided in this paper are with respect to the LSR.

\section{Results}

Our AMBER-MR spectrum shows a rising continuum and a bright $\mathrm{Br} \gamma$ emission line with a PCygni profile (inserts 1a-4a of Fig. 1). No other lines above a three sigma threshold are detected in the spectrum. Figure 1 shows our interferometric observables (line profile - inserts $1 \mathrm{a}-4 \mathrm{a}$; visibilities - inserts $1 \mathrm{~b}$ and $3 \mathrm{~b}$; differential phases - inserts $1 \mathrm{c}$ and $3 \mathrm{c}$, closure phase inserts $1 \mathrm{~d}$ and $3 \mathrm{~d}$ ) of the $\mathrm{Br} \gamma$ line and adjacent continuum for the first (Panel 1) and second run (Panel 3) along with the inferred continuum-corrected $\mathrm{Br} \gamma$-line visibilities (inserts $2 \mathrm{~b}-2 \mathrm{~d}$ and $4 \mathrm{~b}-4 \mathrm{~d}$ ) in four spectral channels (namely those with lineto-continuum ratio larger or equal to 1.1) of run 1 (Panel 2) and run 2 (Panel 4). Visibilities give information on the size of the emitting region (continuum and/or line), whereas the closure phase quantifies its asymmetry. Differential phases are a measure of the photocentre shift of the line with respect to the continuum.

The upper inserts of Fig. 1 (1a-4a) display the $\mathrm{Br} \gamma$ line profile normalised to the continuum. The line is spectrally resolved and shows a wide range of velocities (from 

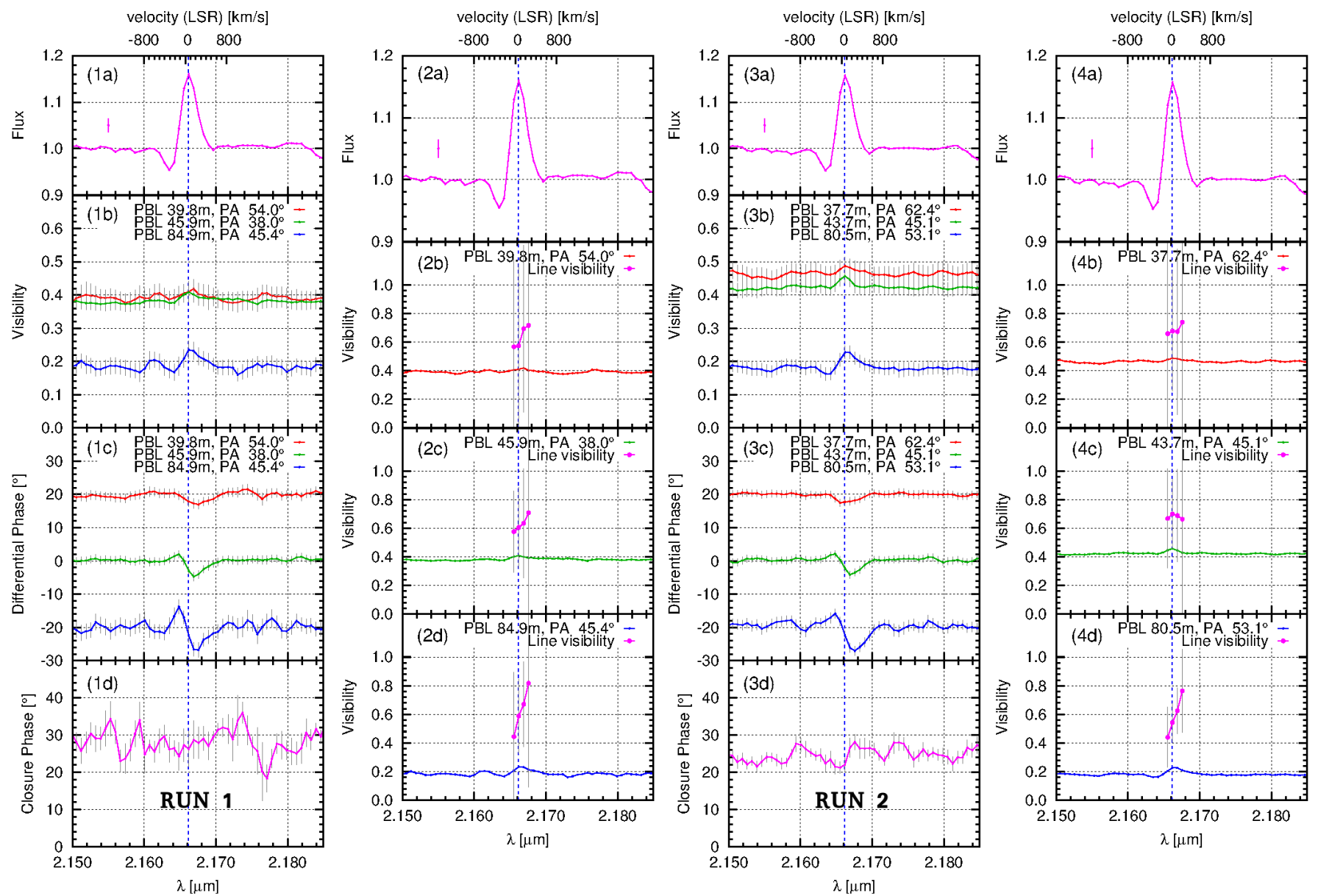

Fig. 1. Left: panel 1. AMBER-MR interferometric measurements of the Bry line in IRAS 13481-6124 for run 1 (inserts 1a-1d). From top to bottom: line flux (1a), wavelength-dependent visibilities (1b), differential phases (1c), and closure phase (1d), observed at three different projected baselines (see labels in figure). For clarity, the differential phases of the first and last baselines are shifted by $+20^{\circ}$ and $-20^{\circ}$, respectively. Middle left: panel2. Visibilities and continuum-corrected (pure) Br $\gamma$-line visibilities of our AMBER-MR observation of IRAS 13481-6124 for run 1 (inserts 2b-2d). From top to bottom: line flux (2a), visibilities of first (2b), second (2c) and third baseline (2d). Middle right: panel 3. AMBER-MR interferometric measurements of the Br $\gamma$ line in IRAS 13481-6124 for run 2 (inserts 3a-3d). Right: panel 4. Visibilities and continuum-corrected (pure) Br $\gamma$-line visibilities of our AMBER-MR observation of IRAS 13481-6124 for run 2 (inserts 4b-4d). Blue dashed lines encompass the emission peak of the $\mathrm{Br} \gamma$ line.

$\sim-500$ to $500 \mathrm{~km} \mathrm{~s}^{-1}$ ). The emission line peaks at about $50 \mathrm{~km} \mathrm{~s}^{-1}$, with a broad red-shifted wing extending up to $\sim 500 \mathrm{~km} \mathrm{~s}^{-1}$. The absorption feature on the blue-shifted side ranges from about -500 to $-150 \mathrm{~km} \mathrm{~s}^{-1}$. The P Cygni profile indicates the presence of outflowing matter in the form of a stellar wind or jet, which produces the typical blue-shifted absorption feature through self-absorption (see, e.g. Mitchell et al. 1991). The outflow terminal velocity is $\sim-500 \mathrm{~km} \mathrm{~s}^{-1}$.

In Fig. 1, upper-middle ( $1 \mathrm{~b}$ and $3 \mathrm{~b}$ ) and lower-middle (1c and 3c) inserts of Panel 1 (left) and Panel 3 (middle-right) show the wavelength-dependent visibilities and differential phases for the three observed baselines of run 1 (Panel 1) and run 2 (Panel 1), respectively, whereas the closure phases are shown in the lower inserts ( $1 b$ and $3 b)$. The results of the two runs are similar although, as mentioned in Sect. 2, the second run data have higher $\mathrm{S} / \mathrm{N}$ ratio. Across the line profile, changes in the visibility and differential phase with respect to the continuum are observed. Blue dashed lines depict the position of the $\mathrm{Br} \gamma-$ emission peak in the four panels.

The $\mathrm{Br} \gamma$ visibility at the line peak is larger than the continuum visibility at all six baselines (see middle-upper panels of left and middle-right inserts in Fig. 1), indicating that, on average, the $\mathrm{Br} \gamma$-emitting region is spatially resolved and more compact than the continuum. The absolute visibilities of the shortest baselines (red and green lines) display higher values, which indicates that both continuum and line are less spatially resolved along these baselines with respect to the longest ones. These short baselines are comparable in length and thus the wavelength-dispersed visibilities are very similar. Notably, the values of the visibility across the $\mathrm{Br} \gamma$-absorption feature at the two longest baselines $\left(85\right.$ and $81 \mathrm{~m}, \mathrm{PA}=45^{\circ}$ and $53^{\circ}$, respectively) are slightly smaller than the average continuum visibility. This means that the $\mathrm{Br} \gamma$ photons at high blue-shifted velocities must originate from a region larger than the continuum, whereas the red-shifted $\mathrm{Br} \gamma$ emission must come from a region that is smaller than the continuum. In other words, the size of the blue-shifted gas in absorption is larger than that of the emitting gas, suggesting the presence of a wind or an outflow.

The differential phase (DP) at the four longest baselines displays an "S" shape, more pronounced at the 85 and $81 \mathrm{~m}$ baselines, whereas the differential phase at the shortest baselines (40 and $38 \mathrm{~m}, \mathrm{PA}=62^{\circ}$ and $54^{\circ}$, respectively) shows a significant displacement with respect to the continuum that appears only close to the line peak and along the red-shifted wing (see middlelower panels of left and middle-right inserts in Fig. 1). This indicates that the outflow is detected mainly at the longest baselines 
and at PAs up to $53^{\circ}$, i.e. that the outflow is quite collimated (within $\sim 27^{\circ}$ from the jet axis, PA $206^{\circ} / 26^{\circ}$ ). Moreover, since the differential phases are related to the line photocentre shifts with respect to the continuum, our findings indicate that both red-shifted and blue-shifted line emission wings, at the longest baselines, are spatially extended and located in opposite directions with respect to the continuum emission.

We note that the observed closure phase (CP; see lower panels of left and middle-right inserts in Fig. 1) differs from zero. Inside the error bars, no significant closure-phase variations of the $\mathrm{Br} \gamma$ line, with respect to the continuum, are detected. This is because of the high noise level in the wavelength differential CPs $\left(\sim 10^{\circ}\right)$, which is about one order of magnitude higher than that in the DPs. The non-zero closure phase originates from the asymmetry of the brightness distribution of the continuum. Owing to the disc inclination of $\sim 45^{\circ}$ with respect to the plane of the sky, the far side of the inner disc rim (i.e. the one towards the blue-shifted side of the jet) displays an area larger (i.e. brighter) than the near side (see paneld of Fig. 1 in Kraus et al. 2010). To confirm this scenario, we compare our observations with the synthetic images around $2.16 \mu \mathrm{m}$ that are derived from the radiative transfer model presented by Kraus et al. (2010). Although this model was originally adjusted to fit AMBER visibilities and closure phases taken with the auxiliary telescopes, it also provides a very good fit to our new, higher SNR UT data. The predicted $\mathrm{CP}$ value of $\sim 26^{\circ}$ is comparable to the measured values of $25^{\circ} \pm 4^{\circ}$ (run 2) and $29^{\circ} \pm 6^{\circ}$ (run 1). Notably the continuum asymmetry also affects the observed DPs of the Br $\gamma$ line, causing the blue-shifted DPs to be systematically smaller than the red-shifted ones.

To infer the size of the Br $\gamma$-emitting region across the different velocity channels, we first compute the continuumsubtracted (or pure-line) visibilities at the six baselines (see middle-left and right inserts in Fig. 1), following Weigelt et al. (2007). The ring-fit diameter of the continuum is taken from Kraus et al. (2010) (5.4 mas or 17.3 au at $3.2 \mathrm{kpc}$ ). The average diameter of the $\mathrm{Br} \gamma$-emitting region (averaged over 8 channels, 4 for each baseline, as plotted for the pure line visibilities of Fig. 1) is $\sim 4$ mas (i. e. $\sim 13$ au at $3.2 \mathrm{kpc}$ ) at the two shortest baselines ( 37.7 and $39.8 \mathrm{~m}), \sim 3.4$ mas $(\sim 11 \mathrm{au})$ at the medium baselines $(43.7$ and $45.9 \mathrm{~m})$, and $\sim 2$ mas $(6.4 \mathrm{au})$ at the longest baselines $(80.5$ and $84.9 \mathrm{~m})$. On average the size of the $\mathrm{Br} \gamma$ emitting region is smaller than the continuum-emitting region. It is worth noting, however, that the PCygni absorption in the blue wing of the $\mathrm{Br} \gamma$ line makes the continuum correction of the visibility uncertain for wavelengths shorter than the peak wavelength of the emission line. In this case, the continuumcorrection overestimates the size of the line-emitting region. This might explain why the values of the pure line visibilities (middle-left and right inserts of Fig. 1) decrease, moving from red-shifted to blue-shifted wavelengths. On the other hand, if this effect is real, it would imply that the two lobes are not spatially symmetric, probably because of the screening effect of the disc on the red-shifted lobe of the outflow.

Finally, the calibrated DPs were converted into photocentre shifts $(p)$ of the $\mathrm{Br} \gamma$-velocity components, with respect to the continuum emission, by solving the following equation: $p_{i}=\left(-D P_{i} \lambda\right) /\left(2 \pi B_{i}\right)$, where $p_{i}$ is the photocentre displacement projection of the $2 \mathrm{D}$ photocentre vector $\boldsymbol{p}$ on the baseline $B_{i}$, and $\lambda$ is the wavelength of the considered spectral channel (Le Bouquin et al. 2009). A single astrometric solution (i.e. a single 2D vector $\boldsymbol{p}$ ) was fitted to all baselines within each spectral channel. As for the visibilities, we then subtracted the continuum contribution to get the photocentre displacements of

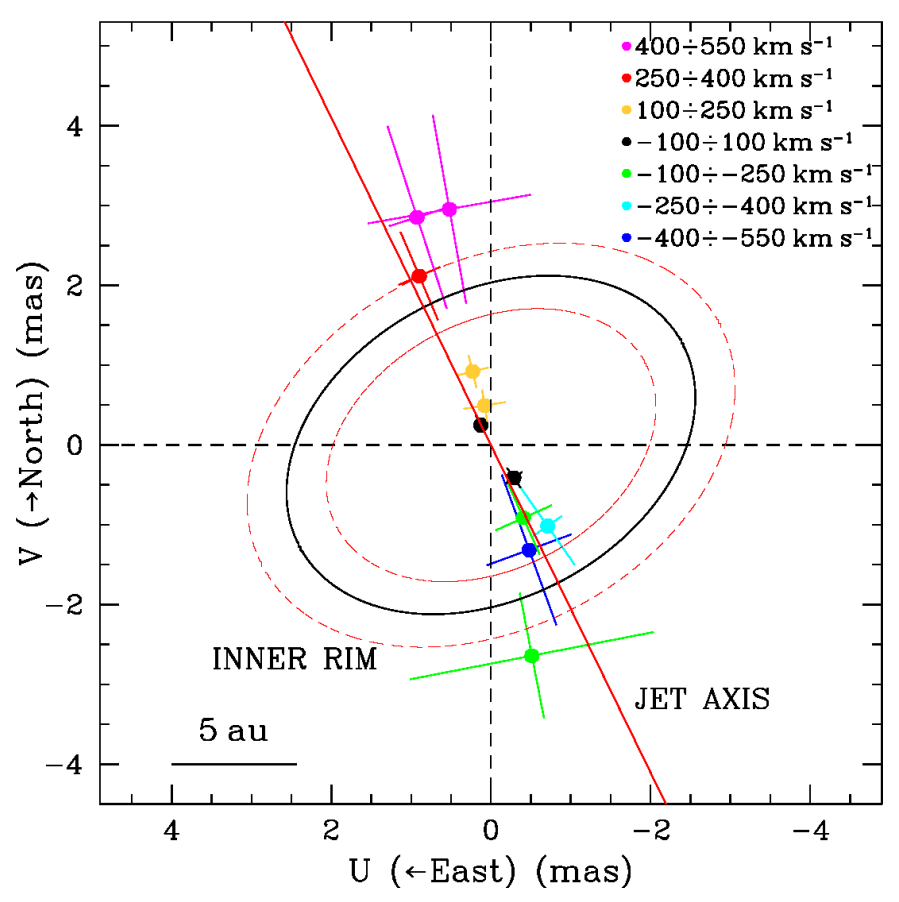

Fig. 2. $\operatorname{Br} \gamma$ displacement for different (radial) velocity channels (with velocity bins from -550 to $550 \mathrm{~km} \mathrm{~s}^{-1}$ ) derived from the measured differential phases, after correcting for the continuum contribution. Different colours indicate different velocity bins. Black solid and red dashed ellipses indicate the extent of the $K$-band continuum with its uncertainty $(1.94 \pm 0.37$ mas Kraus et al. 2010), whereas the red line shows the position of the jet axis (Caratti o Garatti et al. 2015a).

the pure-line emitting region (see Eq. (3) in Kraus et al. 2012). Figure 2 shows the continuum-subtracted $\mathrm{Br} \gamma$-line photocentre displacements (with the errorbars in polar coordinates), which were derived from the astrometric solution in the different (radial) velocity channels (colour coded). Both the inner rim, defined by the study of the NIR continuum (see Kraus et al. 2010), and the known jet axis (see Caratti o Garatti et al. 2015a), are shown as a black solid ellipse and red solid line, respectively. The red dashed ellipses indicate the uncertainty on the size of the inner rim (1.97 \pm 0.37 mas; Kraus et al. 2010). Figure 2 shows that the photocentre-shifts in the blue-shifted (green, cyan and blue dots; from $v_{\mathrm{r}} \sim-420$ to $\sim-140 \mathrm{~km} \mathrm{~s}^{-1}$ ) and red-shifted (orange, red, and magenta dots; from $v_{\mathrm{r}} \sim 140$ to $\sim 520 \mathrm{~km} \mathrm{~s}^{-1}$ ) wings of the $\mathrm{Br} \gamma$ are increasing considerably with increasing velocities and are roughly displaced along a straight line, close to the jet axis, in the blue- and red-shifted lobes, respectively. The significance of the red-shifted data points at the highest velocity (magenta dots) is reduced with respect to the other points since they are seen out of the obscuring disc only because of the large uncertainty of their position.

The low-velocity components (black dots; with $v_{\mathrm{r}} \sim-50$ and $50 \mathrm{~km} \mathrm{~s}^{-1}$ ) are also aligned with the jet axis, but are much closer to the central source $(\lesssim 2 \mathrm{au})$, indicating a strong acceleration of the gas in the inner $10 \mathrm{au}$. We note that, at $2 \mathrm{au}$, the Keplerian velocity of a $20 M_{\odot}$ object is $\sim 95 \mathrm{~km} \mathrm{~s}^{-1}$ ( or $v_{\mathrm{r}} \sim 67 \mathrm{~km} \mathrm{~s}^{-1}$ ), that is, it is not spectrally resolved by our observations $(\Delta v \sim$ $140 \mathrm{~km} \mathrm{~s}^{-1}$ ). Our findings thus suggest that, on the one hand, the high-velocity component is tracing the jet/outflow, and, on the other, the low-velocity component is tracing slow moving gas, such as a disc wind (in Keplerian rotation) or the very base of the jet, namely the region of the disc where the jet footpoints are located. 


\section{Origin of the $\mathrm{Br} \gamma$ line in IRAS 13481-6124}

The origin of the $\mathrm{Br} \gamma$ line in YSOs is controversial and there are several physical mechanisms that could produce this emission, such as accretion of matter onto the star (e.g. Eisner et al. 2009), or outflowing material from a disc wind (e.g. Weigelt et al. 2011; Garcia Lopez et al. 2015; Caratti o Garatti et al. 2015b), extended wind or outflow (e.g. Garcia Lopez et al. 2016), or jet (e.g. Stecklum et al. 2012).

Our AMBER/VLTI observations of IRAS 13481-6124 suggest that the main $\mathrm{Br} \gamma$ emission emanates from an ionised jet. The observed $\mathrm{Br} \gamma$ PCygni profile indicates the presence of a fast ionised jet/outflow $\left(\sim 500 \mathrm{~km} \mathrm{~s}^{-1}\right)$. This value agrees well with the radio jet velocities measured in other HMYSOs (see, e.g. Heathcote et al. 1998; Martí et al. 1998; Curiel et al. 2006; Torrelles et al. 2011). Visibilities and differential phases at high velocities indicate that the outflowing matter is spatially extended, from a few au to a few tens of au, and the photocentre shifts grow with increasing radial velocities. Both observables also suggest that the flow is relatively well collimated $\left(\lesssim 30^{\circ}\right)$ close to the known parsec-scale jet PA, implying that we are observing a collimated wind or flow. Initial jet opening angles are typically $\sim 30^{\circ}$ in YSOs and a considerable degree of focusing in low-mass YSOs happens at several au from the source (see, e.g. Ray et al. 2007). It is thus reasonable to presume that the corresponding jet collimation in HMYSOs occurs at even larger distances from the source (a few tens of au). Indeed, as magneto-centrifugal launching models are scale-free (see, e.g. Ferreira 1997; Ferreira \& Casse 2004; Pudritz et al. 2007), for higher masses the configuration readjusts to larger scales consistently with the different values of the gravitational potential. As IRAS 13481-6124 has a parsec-scale jet, we could then argue that the high-velocity component of the $\operatorname{Br} \gamma$ line $\left(500<v_{\mathrm{r}}\right.$ $<100 \mathrm{~km} \mathrm{~s}^{-1}$ ) is tracing the (poorly collimated) jet, which extends from a few au to tens of au from the central source. On the other hand, the low-velocity component $\left(v_{\mathrm{r}}<100 \mathrm{~km} \mathrm{~s}^{-1}\right)$, although partially resolved, is more compact, being located $\lesssim 2$ au from the source and well inside the inner-rim disc. This slow moving gas component is also aligned with the jet axis. Owing to our limited spectral resolution and given that this velocity is also compatible with Keplerian rotation (at a distance of $\lesssim 2 \mathrm{au}$ ), this component might originate from a disc wind or the jet footpoint. We note that a similar geometry for the $\mathrm{Br} \gamma$ emission in another HMYSO (W 33A) was inferred by Davies et al. (2010) with spectro-astrometry. Finally, even if the error bars are considered, the $\mathrm{Br} \gamma$ visibility is lower than one, i.e. it is spatially resolved at the longest baselines, indicating that the bulk of the emission cannot originate from accretion, which would not be spatially resolved at our baselines. Moreover, infall gas would have free-fall velocities of several hundreds of $\mathrm{km} \mathrm{s}^{-1}$, which are not detected close to the source.

In conclusion, most of the observed $\mathrm{Br} \gamma$ emission must originate from the ionised jet. In principle, it should be fully ionised because of being exposed to the UV radiation of a $20 M_{\odot}$ HMYSO (Tanaka et al. 2016). As for the case of irradiated jets in massive star-forming regions (see, e.g. Reipurth et al. 1998;
Bally et al. 2006), the gas of the jet would then be fully traced by the HI emission and its denisty proportional to the HI line intensity. Therefore, measurements of intensity, velocity, and size of the Br $\gamma$-emitting region might provide a good estimate of the mass-loss rate.

Acknowledgements. A.C.G., R.G.L., and T.P.R. were supported by Science Foundation Ireland, grant 13/ERC/I2907. A.K. and S.K. acknowledge support from a STFC Ernest Rutherford fellowship and grant (ST/J004030/1, ST/K003445/1), and Marie-Sklodowska Curie CIG grant (Ref. 618910). A.S. was supported by the Deutsche Forschungsgemeinschaft (DFG) Priority Program 1573. This research has also made use of NASA's Astrophysics Data System Bibliographic Services and the SIMBAD database operated at the CDS, Strasbourg, France.

\section{References}

Bally, J., Licht, D., Smith, N., \& Walawender, J. 2006, AJ, 131, 473 Boley, P. A., Kraus, S., de Wit, W.-J., et al. 2016, A\&A, 586, A78 Caratti o Garatti, A., Stecklum, B., Linz, H., Garcia Lopez, R., \& Sanna, A. 2015a, A\&A, 573, A82

Caratti o Garatti, A., Tambovtseva, L. V., Garcia Lopez, R., et al. 2015b, A\&A, 582, A44

Cesaroni, R., Galli, D., Neri, R., \& Walmsley, C. M. 2014, A\&A, 566, A73

Curiel, S., Ho, P. T. P., Patel, N. A., et al. 2006, ApJ, 638, 878

Davies, B., Lumsden, S. L., Hoare, M. G., Oudmaijer, R. D., \& de Wit, W.-J. 2010, MNRAS, 402, 1504

Eisner, J. A., Graham, J. R., Akeson, R. L., \& Najita, J. 2009, ApJ, 692, 309

Ferreira, J. 1997, A\&A, 319, 340

Ferreira, J., \& Casse, F. 2004, Ap\&SS, 292, 479

Gai, M., Menardi, S., Cesare, S., et al. 2004, in New Frontiers in Stellar Interferometry, ed. W. A. Traub, SPIE Conf. Ser., 5491, 528

Garcia Lopez, R., Tambovtseva, L. V., Schertl, D., et al. 2015, A\&A, 576, A84 Garcia Lopez, R., Kurosawa, R., Caratti o Garatti, A., et al. 2016, MNRAS, 456, 156

Grave, J. M. C., \& Kumar, M. S. N. 2009, A\&A, 498, 147

Heathcote, S., Reipurth, B., \& Raga, A. C. 1998, AJ, 116, 1940

Ilee, J. D., Wheelwright, H. E., Oudmaijer, R. D., et al. 2013, MNRAS, 429, 2960

Königl, A., \& Pudritz, R. E. 2000, Protostars and Planets IV, 759

Kraus, S., Hofmann, K.-H., Menten, K. M., et al. 2010, Nature, 466, 339

Kraus, S., Monnier, J. D., Che, X., et al. 2012, ApJ, 744, 19

Le Bouquin, J.-B., Absil, O., Benisty, M., et al. 2009, A\&A, 498, L41

Lumsden, S. L., Hoare, M. G., Urquhart, J. S., et al. 2013, ApJS, 208, 11

Martí, J., Rodríguez, L. F., \& Reipurth, B. 1998, ApJ, 502, 337

Mitchell, G. F., Maillard, J.-P., \& Hasegawa, T. I. 1991, ApJ, 371, 342

Petrov, R. G., Malbet, F., Weigelt, G., et al. 2007, A\&A, 464, 1

Pudritz, R. E., Ouyed, R., Fendt, C., \& Brandenburg, A. 2007, in Protostars and Planets V, eds. B. Reipurth, D. Jewitt, \& K. Keil, 277

Ray, T., Dougados, C., Bacciotti, F., Eislöffel, J., \& Chrysostomou, A. 2007, in Protostars and Planets V, eds. B. Reipurth, D. Jewitt, \& K. Keil, 231

Reipurth, B., Bally, J., Fesen, R. A., \& Devine, D. 1998, Nature, 396, 343

Sanna, A., Cesaroni, R., Moscadelli, L., et al. 2014, A\&A, 565, A34

Sanna, A., Surcis, G., Moscadelli, L., et al. 2015, A\&A, 583, L3

Stecklum, B., Caratti o Garatti, A., Davis, C., et al. 2009, Astrophys. Space Sci. Proc., 13, 619

Stecklum, B., Caratti o Garatti, A., \& Linz, H. 2012, in Circumstellar Dynamics at High Resolution, eds. A. C. Carciofi, \& T. Rivinius, ASP Conf. Ser., 464, 369

Tan, J. C., Beltrán, M. T., Caselli, P., et al. 2014, Protostars and Planets VI, 149

Tanaka, K. E. I., Tan, J. C., \& Zhang, Y. 2016, ApJ, 818, 52

Tatulli, E., Millour, F., Chelli, A., et al. 2007, A\&A, 464, 29

Torrelles, J. M., Patel, N. A., Curiel, S., et al. 2011, MNRAS, 410, 627

Varricatt, W. P., Davis, C. J., Ramsay, S., \& Todd, S. P. 2010, MNRAS, 404, 661

Weigelt, G., Kraus, S., Driebe, T., et al. 2007, A\&A, 464, 87

Weigelt, G., Grinin, V. P., Groh, J. H., et al. 2011, A\&A, 527, A103 